\title{
HABILIDADES DE PRAXIA VERBAL E NÃO-VERBAL EM INDIVÍDUOS GAGOS
}

\author{
Verbal and non-verbal praxic abilities in stutterers
}

\author{
Natália Casagrande Brabo ${ }^{(1)}$, Ana Maria Schiefer ${ }^{(2)}$
}

\begin{abstract}
RESUMO
Objetivo: caracterizar as habilidades de praxias verbal e não-verbal em indivíduos gagos. Métodos: participaram do estudo 40 indivíduos, com idade igual ou superior a 18 anos, do sexo masculino e feminino: 20 gagos adultos e 20 sem queixas de comunicação. Para a avaliação das praxias verbal e não-verbal, os indivíduos foram submetidos à aplicação do Protocolo de Avaliação da Apraxia Verbal e Não-verbal (Martins e Ortiz, 2004). Resultados: com relação às habilidades de praxia verbal houve diferença estatisticamente significante no número de disfluências típicas e atípicas apresentadas pelos grupos estudados. Quanto à tipologia das disfluências observou-se que nas típicas houve diferença estatisticamente significante entre os grupos estudados apenas na repetição de frase, e nas atípicas, houve diferença estatisticamente significante, tanto no bloqueio quanto na repetição de sílaba e no prolongamento. Com relação às habilidades de praxia não-verbal, não foram observadas diferenças estatisticamente significantes entre os indivíduos estudados na realização dos movimentos de lábios, língua e mandíbula, isolados e em sequência. Conclusão: com relação às habilidades de praxia verbal, os gagos apresentaram frequência maior de rupturas da fala, tanto de disfluências típicas quanto de atípicas, quando comparado ao grupo controle. Já na realização de movimentos práxicos isolados e em sequência, ou seja, nas habilidades de praxia não-verbal, os indivíduos gagos não se diferenciaram dos fluentes não confirmando a hipótese de que o início precoce da gagueira poderia comprometer as habilidades de praxia não-verbal.
\end{abstract}

DESCRITORES: Gagueira; Distúrbios da Fala; Apraxias

\section{INTRODUÇÃO}

A fluência envolvida na sequência dos movimentos exigidos para a fala é denominada de praxia verbal ou praxia de fala. Em outras palavras, a praxia de fala é a capacidade de sequencialização das sílabas nas palavras, sem hesitações, e depende do amadurecimento da zona pré-motora da linguagem e suas conexões, o que ocorre em torno dos 2 anos, quando a criança emite palavras e constrói frases agramaticais. Nessa época é comum aparecerem também as disfluências fisiológicas e, é de

(1) Fonoaudióloga; Clínica Brabo, São Paulo, SP; Especialista em Distúrbios da Comunicação Humana pela Universidade Federal de São Paulo.

(2) Fonoaudióloga; Professora Adjunta do Departamento de Fonoaudiologia da Universidade Federal de São Paulo, UNIFESP, São Paulo, SP; Doutora em Ciências dos Distúrbios da Comunicação Humana pela Universidade Federal de São Paulo.

Conflito de interesses: inexistente consenso, que (80\%) da gagueira desenvolvimental tem início neste período.

A gagueira desenvolvimental é uma desordem de comunicação que começa na infância ${ }^{1}$, e é caracterizada por perturbações involuntárias na fluência da expressão verbal. Os comportamentos mais típicos são as repetições de sons ou sílabas, bloqueios e prolongamento de sons. Já foi reconhecido que há um componente genético responsável pela desordem de fala - gagueira e que aproximadamente metade dos gagos apresentam uma história da desordem na família ${ }^{1,2}$, no entanto, o modo exato de transmissão ainda é desconhecido ${ }^{3}$.

A fala é uma tarefa de controle motor rápido, em que os movimentos devem ocorrer em poucos milissegundos; esse rápido processamento requer uma alta resolução temporal ${ }^{4}$. A fala fluente é a capacidade de conversar com continuidade a uma velocidade adequada e sem esforço. Indivíduos com gagueira do desenvolvimento apresentam uma perturbação crônica na capacidade de produzir a fala 
de forma fácil e contínua, já que o processamento neurofisiológico da fala fluente depende da estabilidade da coordenação temporal entre as habilidades de execução motora e representação do processamento cognitivo ${ }^{5}$. Assim, a disfluência é um distúrbio que ocorre no momento da produção da fala, na qual o falante se depara com um impedimento que o impossibilita, momentaneamente, de produzir a palavra que deseja falar ${ }^{6}$.

Já as praxias do sistema estomatognático prejudicam a mímica facial e a imitação de gestos de lábios e de língua, podendo resultar em dificuldades articulatórias da fala. A apraxia oral é uma desordem na qual a criança, que tipicamente começou a falar tarde, é incapaz de coordenar ou iniciar movimentos de mandíbula, lábios e língua sob comando ${ }^{7}$. É de consenso, que as crianças não nascem com a praxia totalmente desenvolvida. Esta é considerada um aprendizado funcional, não apenas um produto da maturação motora, mas que requer interação com a produção da fala. As habilidades práxicas se desenvolvem num contínuo ordenado dos 02 aos 12 anos de idade. A ordem deste desenvolvimento é mantida por evidências neurológicas baseada na maturação cerebral ${ }^{8}$.

Além disso, sabe-se que na habilidade práxica não-verbal, os movimentos de lábios, língua e mandíbula sofrem modificações e, os movimentos indiferenciados no início da infância, passam a ser refinados e diferenciados conforme o desenvolvimento ${ }^{9}$. Os indivíduos com gagueira são capazes de utilizar a estrutura oral sem dificuldades para as atividades sequenciais que não envolvem a fala, como por exemplo, durante a mastigação, sucção, deglutição. Pela quebra súbita dos padrões, o déficit neuromuscular só ocorre quando é requerida uma coordenação suave e rápida para a fala, ou seja, para o ajustamento preciso dos articuladores (língua, mandíbula e lábios) numa organização estruturalmente rítmica ${ }^{10}$.

Assim, considerando o embricamento destas questões o presente estudo tem por objetivo caracterizar as habilidades de praxias verbal e não-verbal em indivíduos gagos.

\section{MÉTODOS}

Esta pesquisa foi realizada no Ambulatório de Avaliação e Diagnóstico dos Distúrbios da Comunicação Humana, da Universidade Federal de São Paulo, UNIFESP, durante o período de 2007.

Foram selecionados 40 indivíduos, sendo 20 gagos (Grupo I) e 20 não gagos (Grupo II), do sexo masculino e feminino, com idade igual ou superior a 18 anos. Para o grupo de gagos foram selecionados apenas indivíduos que apresentavam no mínimo 3\% de disfluências atípicas na fala, ou seja, aqueles que poderiam ser classificados como gagos, segundo a literatura ${ }^{11,12}$. Foram excluídos deste grupo aqueles que apresentaram menos de $3 \%$ de disfluências atípicas na fala, por se constituírem, provavelmente, de casos considerados limítrofes ou naturais, e aqueles com outros comprometimentos associados - neurológico, auditivo, visual e/ ou emocional. Além disso, foram excluídos aqueles indivíduos com alteração de tônus e mobilidade dos órgãos fonoarticulatórios, já que estas alterações poderiam interferir diretamente nos resultados da pesquisa.

Para confirmação do diagnóstico de gagueira, todos os participantes da pesquisa foram submetidos à avaliação fonoaudiológica completa através da qual foram coletadas informações da anamnese, principalmente aquelas relacionadas à queixa e a história do desenvolvimento da gagueira e das habilidades comunicativas dos indivíduos. Da avaliação específica da fala foram coletadas informações sobre a tipologia, a frequência das disfluências, e o nível de severidade da gagueira.

Para o grupo controle foram selecionados indivíduos sem queixas de comunicação, que apresentaram disfluências consideradas dentro dos limites de normalidade, ou seja, menos de 3\% de disfluências atípicas e menos $10 \%$ de disfluências típicas, verificadas através de uma coleta da fala encadeada.

Para coleta dos dados foram utilizados critérios da literatura que classificam as disfluências em típicas e atípicas ${ }^{11,12}$. Como disfluências típicas foram consideradas as hesitações, interjeições, revisões, palavras incompletas, repetições de frases e de palavras (até duas vezes), e como disfluências atípicas, as repetições de palavras (três ou mais vezes), de sílabas e de sons, bloqueios, pausas longas e intrusão.

Para a avaliação do grau de severidade da gagueira foi utilizado o protocolo S.S.I-3 Stuttering Severity Instrument ${ }^{11}$, que considera a frequência e duração das rupturas da fala, bem como a presença de movimentos associados a essas rupturas, por meio da análise da fala espontânea e da leitura.

Para a avaliação das praxias foi utilizado o Protocolo de Avaliação da Apraxia de Fala ${ }^{13}$. As provas para avaliação da praxia verbal constituíram-se de: tarefas de repetição de palavras e sentenças, fala espontânea, automatismos e leitura em voz alta de palavras e sentenças. Na tarefa de repetição de palavras e sentenças, os estímulos foram lidos para que os indivíduos pudessem repetir. $\mathrm{Na}$ fala espontânea foi solicitado aos indivíduos que descrevessem uma figura de um determinado cartão temático, nos automatismos, que contassem números de 1 a 20 e dissessem os meses do ano, na 
leitura em voz alta de palavras e frases, que lessem os estímulos. As disfluências presentes na fala dos indivíduos foram mapeadas e transcritas pela avaliadora através das amostras de fala coletadas.

Já, as provas para a avaliação da praxia nãoverbal constituíram-se de 20 movimentos; isolados (mostrar os dentes, mostrar o sorriso, protruir a língua, assoprar, elevar a língua, morder o lábio inferior, pigarrear, abaixar a língua, fazer um bico, tossir, inflar as bochechas, mandar um beijo, lateralizar a mandíbula, passar a língua nos lábios e estalar a língua) e em sequência (protruir e retrair a língua, lateralizar a língua, elevar e abaixar a língua, alternar bico e sorriso, lateralizar e elevar a língua), executados pelos indivíduos de ambos os grupos, após um comando verbal. Em situações na qual o comando verbal não foi suficiente para a execução correta do movimento, este foi demonstrado pela avaliadora a fim de facilitar o desempenho através da pista visual, e então classificado de acordo com uma escala gradual de respostas: 160 e 200 pontos foram considerados normais, 80 e 159 apráxicos não-verbais leves, 40 e 79 apráxicos moderados e, os que não ultrapassaram 39 pontos apráxicos graves. Alem disso, a avaliação da sequência dos movimentos de lábios e língua foi realizada durante 5 segundos ${ }^{7}$.

A coleta dos dados foi realizada individualmente e gravada em câmera digital (SONY Cyber - shot 6.0 mega pixels).

O estudo foi iniciado após aprovação pelo Comitê de Ética em Pesquisa da instituição (protocolo no 1106/07), e a coleta dos dados após a autorização dos participantes, obtida por meio do Termo de Consentimento Livre e Esclarecido.

Para a análise dos dados referentes às habilidades de praxia verbal foi utilizado o teste não paramétrico de Mann-Whitney, e das habilidades de praxia não-verbal o Teste Exato de Fisher, e considerado um nível de significância de 5\%.

\section{RESULTADOS}

Os indivíduos gagos apresentaram a seguinte distribuição: sexo (11 feminino, 9 masculino), idade (média de 24,1 anos), e grau de severidade (55\% de grau leve, $15 \%$ moderado, $10 \%$ severo e $20 \%$ muito severo), e foram pareados por sexo e idade (media 24, 6 anos) aos indivíduos do grupo controle.

A distribuição das disfluências típicas e atípicas são apresentadas nas Tabelas 1 e 2. Na avaliação das habilidades de praxia verbal observou-se disfluências na fala encadeada (média 200 sílabas), nos indivíduos do grupo de gagos e não gagos, a distribuição das disfluências típicas e atípicas foi mais acentuada no grupo de indivíduos gagos quando comparada ao grupo de não gagos, como se pode observar nas Tabelas 1 e 2 .

Houve também uma diferenciação quanto à distribuição dessas rupturas segundo a tipologia. Quanto à disfluência típica, pode-se observar que houve diferença estatisticamente significante entre os grupos estudados apenas na disfluências do tipo repetição de frase. Para os demais tipos de disfluências típicas observadas (interjeição, hesitação, repetição de palavra) não foram encontradas diferenças entre os grupos.

Com relação às disfluências atípicas (Tabela 2) houve diferença estatisticamente significante, principalmente nas disfluências do tipo bloqueio, prolongamento e, repetição de sílabas, entre os grupos estudados, ou seja, o Gl apresentou número maior de disfluencias comparado ao Gll.

Nas Tabelas 3 e 4, encontram-se os resultados da avaliação das habilidades de praxia não-verbal nos indivíduos gagos e não gagos. Observou-se pontuação dentro dos parâmetros de normalidade para ambos os grupos.

\section{DISCUSSÃO}

É de consenso em estudos na literatura, que a frequência das disfluências típicas e atípicas é acentuada em grupos de indivíduos gagos quando comparada aos grupos de não gagos, pois estas manifestações constituem-se como características principais da gagueira ${ }^{11,12}$. Neste estudo, como se pode observar nas Tabelas 1 e 2, os resultados encontrados nas provas de praxia verbal corroboram os achados da literatura, pois o grupo de gagos apresentou maior número de disfluências típicas e atípicas quando comparado aos indivíduos do grupo controle.

Com relação às disfluências típicas e atípicas, em estudos semelhantes, realizados com indivíduos gagos e não gagos, em diferentes idiomas, observou-se que a diferenciação quanto à distribuição das rupturas (típicas e atípicas) foi bastante variável ${ }^{11,12,14-16}$.

Quanto ao desempenho dos indivíduos gagos e não gagos, nas provas de praxia não-verbal, com movimentos isolados e em sequência, observa-se que ambos apresentaram pontuação dentro dos parâmetros de normalidade. Entretanto, a partir da comparação do desempenho nas provas de praxia não-verbal dos indivíduos de ambos os grupos, verificadas na Tabela 3 e 4, pode-se supor que a possibilidade de um indivíduo gago ser classificado como não adequado é maior do que a de um indivíduo não gago, tanto para a realização de movimentos isolados como em sequência. 
Tabela 1 - Distribuição de disfluências típicas apresentadas pelos indivíduos de cada grupo (gagos e controle)

\begin{tabular}{|c|c|c|c|c|c|c|c|c|c|c|c|c|c|c|}
\hline & \multicolumn{3}{|c|}{ Interjeição } & \multicolumn{3}{|c|}{ Hesitação } & \multicolumn{3}{|c|}{ Rep. Frase } & \multicolumn{3}{|c|}{ Rep. Palavra } & \multicolumn{2}{|c|}{ Total } \\
\hline & $\mathbf{T}$ & $M$ & $\%$ & $\mathbf{T}$ & $M$ & $\%$ & $\mathbf{T}$ & M & $\%$ & $\mathbf{T}$ & M & $\%$ & $\mathbf{T}$ & $\%$ \\
\hline $\mathrm{Gl}$ & 7 & 0 & 13,46 & 12 & 0 & 23,07 & 16 & 0,5 & 30,76 & 17 & 0 & 32,69 & 52 & 100 \\
\hline GII & 2 & 0 & 11,11 & 10 & 0 & 55,55 & 2 & 0 & 11,11 & 4 & 0 & 22,2 & 18 & 100 \\
\hline Estatística W & & 379 & & & 403 & & & 326 & & & 362 & & & \\
\hline P- Valor & & 0,1056 & & & 0,419 & & & 0,0025 & & & 0,0554 & & & \\
\hline
\end{tabular}

Legenda: T- total; M- mediana;\% - porcentagem ; Pal- palavra; Rep- repetição

Tabela 2 - Distribuição de disfluências atípicas apresentadas pelos indivíduos de cada grupo (gagos e controle)

\begin{tabular}{|c|c|c|c|c|c|c|c|c|c|c|c|c|c|c|c|c|c|}
\hline & \multicolumn{3}{|c|}{ Bloqueio } & \multicolumn{3}{|c|}{ Prolongamento } & \multicolumn{3}{|c|}{ Rep. Sílaba } & \multicolumn{3}{|c|}{ Rep. Som } & \multicolumn{3}{|c|}{ Intrusão } & \multicolumn{2}{|c|}{ Total } \\
\hline & $T$ & M & $\%$ & $\mathbf{T}$ & $M$ & $\%$ & $\mathbf{T}$ & M & $\%$ & $T$ & $M$ & $\%$ & $T$ & M & $\%$ & $T$ & $\%$ \\
\hline GI & 337 & 6 & 70,06 & 61 & 1 & 12,68 & 68 & 1,5 & 14,13 & 6 & 0 & 1,24 & 4 & $\begin{array}{c}\text { não } \\
\text { foi }\end{array}$ & 1,89 & 481 & 100 \\
\hline GII & 1 & 0 & 10 & 6 & 0 & 60 & 2 & 0 & 20 & 1 & 0 & 10 & 0 & $\begin{array}{l}\text { pos- } \\
\text { Sível }\end{array}$ & & 10 & 100 \\
\hline Estatística W & & 233 & & & 292 & & & 290 & & & 389 & & & $\begin{array}{l}\text { reali- } \\
\text { zação } \\
\text { de }\end{array}$ & & & \\
\hline P- Valor & & 0 & & & 0,003 & & & 0,001 & & & 0,1434 & & & s estatí & icos & & \\
\hline
\end{tabular}

Legenda: T- total; M- mediana; \% - porcentagem ; Rep- repetição

Tabela 3 - Comparação do desempenho nas provas de praxia não-verbal dos indivíduos dos grupos de gagos e controle, com movimentos isolados

\begin{tabular}{|c|c|c|c|c|c|c|}
\hline & \multicolumn{2}{|c|}{ Não Adequado } & \multicolumn{2}{|c|}{ Adequado } & \multicolumn{2}{|c|}{ Total } \\
\hline & $\mathbf{N}$ & $\%$ & $\mathrm{~N}$ & $\%$ & $\mathbf{N}$ & $\%$ \\
\hline Gl & 16 & 72,73 & 4 & 22,23 & 20 & 50 \\
\hline GII & 6 & 27,27 & 14 & 77,77 & 20 & 50 \\
\hline Total & 22 & 100 & 18 & 100 & 40 & 100 \\
\hline Razão de chace estimada & \multicolumn{6}{|c|}{8,723} \\
\hline P. Valor & \multicolumn{6}{|c|}{0,001} \\
\hline
\end{tabular}

Tabela 4 - Comparação do desempenho dos indivíduos dos grupos de gagos e controle, nas provas de praxia não-verbal com movimentos em sequência

\begin{tabular}{|c|c|c|c|c|c|c|c|c|c|c|}
\hline & \multicolumn{2}{|c|}{$\begin{array}{c}\text { Protruir e retrair a } \\
\text { língua }\end{array}$} & \multicolumn{2}{|c|}{ Lateralizar a língua } & \multicolumn{2}{|c|}{$\begin{array}{c}\text { Elevar e abaixar a } \\
\text { língua }\end{array}$} & \multicolumn{2}{|c|}{$\begin{array}{c}\text { Alternar } \\
\text { Bico/sorriso }\end{array}$} & \multicolumn{2}{|c|}{$\begin{array}{l}\text { Lateralizar e elevar } \\
\text { a língua }\end{array}$} \\
\hline & NA & A & NA & A & NA & A & NA & A & NA & A \\
\hline & $\%$ & $\%$ & $\%$ & $\%$ & $\%$ & $\%$ & $\%$ & $\%$ & $\%$ & $\%$ \\
\hline GI & 33,4 & 51,34 & 66,6 & 48,65 & 75 & 43,75 & 80 & 45,71 & 75 & 47,23 \\
\hline Gll & 66,6 & 48,65 & 33,4 & 51,35 & 25 & 56,25 & 20 & 54,29 & 25 & 52,77 \\
\hline Total & 100 & 100 & 100 & 100 & 100 & 100 & 100 & 100 & 100 & 100 \\
\hline P- Valor & \multicolumn{2}{|c|}{1} & \multicolumn{2}{|c|}{1} & \multicolumn{2}{|c|}{0,235} & \multicolumn{2}{|c|}{0,341} & \multicolumn{2}{|c|}{0,605} \\
\hline
\end{tabular}

Legenda: NA- não adequado; A- adequado

Anomalias no controle de movimentos orais, já foram identificadas em gagos, o que sugere que esta desordem de fala envolve um déficit sensóriomotor. Estudos realizados com adultos gagos e fluentes, na realização de uma tarefa de abertura de mandíbula, e abertura de mandíbula associada à fonação, encontraram uma diferença sutil de integração proprioceptiva nos indivíduos com gagueira, 
sugerindo que o uso da propriocepção em gagos é menos eficiente que nos oradores fluentes, que apresentam melhor integração sensório-motora durante produção da fala ${ }^{17}$, ou seja, a gagueira pode estar associada a uma limitação de propriocepção oral, embora, os resultados também sejam coerentes com um déficit de controle de motor ${ }^{18}$.

As informações da percepção oral são processadas durante a produção de fala e são necessárias para o controle dos movimentos de fala a longo prazo ${ }^{19}$. Neste contexto, é significativo que adultos que gaguejam apresentem anomalias na percepção oral ${ }^{18}$.

A fala envolve a coordenação de múltiplos articuladores - lábios, mandíbula, língua e laringe ${ }^{20}$. A integração sensório-motora deficiente em gagos evidencia um déficit de propriocepção, porém, vários estudos nesta área ainda consideram este assunto um desafio, já que os resultados destes diferem de outros ${ }^{21,22}$. Os estudos evidenciam uma deficiência sutil na integração sensório-motora oral, que afeta a coordenação motora durante produção da fala e também durante as tarefas não-verbais.

A gagueira pode envolver um déficit sensóriomotor, mas, claramente outros fatores tais como: desenvolvimento de linguagem, complexidade linguística e diferenças individuais fornecem evidências que outros fatores devem ser considerados ${ }^{23}$.

Estudos propõem, que um déficit sensório-motor oral faz com que a criança que gagueja torne-se mais vulnerável a desenvolver gagueira crônica, enquanto também impede a eficácia de tratamento. Em estudos futuros, tarefas de integração sensóriomotoras orais necessitam ser adaptadas para testar crianças e verificar se estas alterações são uma característica de indivíduos que vão manifestar a gagueira crônica ${ }^{24,25}$.

Outros estudos de produção da fala não identificaram diferenças entre oradores gagos e fluentes ${ }^{26}$. A gagueira pode ser caracterizada em parte como uma desordem na coordenação de diferentes sistemas de músculos, porém, as diferenças entre gagos e não gagos não estão confinadas apenas aos processos sensório-motores subjacentes à produção da fala nem mesmo a movimentos do sistema de orofacial em geral ${ }^{27}$.

Estudo realizado com eletromiografia, concluiu que os indivíduos gagos não apresentam níveis maiores de atividade muscular de lábio quando comparados com oradores fluentes ${ }^{28}$. Entretanto, em estudos realizados com crianças com distúrbios articulatórios, e crianças com distúrbios articulatórios associados à gagueira, foi observado desempenho pior em provas de praxia oral, sendo que muitas delas apresentaram menor dificuldade em movimentos isolados quando comparados aos movimentos em sequência ${ }^{29}$. Por outro lado, em estudo realizado com crianças pré-escolares não foi observada relação entre fala, tônus e praxia nãoverbal ${ }^{30}$.

Embora os resultados das pesquisas sobre neurofisiologia do processamento da fala fluente $e$ disfluente ainda não apresentem resultados conclusivos, os estudos realizados em larga escala confirmam que, nos padrões de ativação neural, existe uma diferença significativa entre adultos gagos e fluentes durante a fala e essa diferença existe mesmo quando não há movimento articulatório ${ }^{31-35}$.

\section{CONCLUSÃO}

Com relação às rupturas da fala, tanto as disfluências típicas quanto as atípicas foram mais acentuadas nos indivíduos gagos quando comparados aos do grupo controle, como seria esperado.

Já, na realização dos movimentos práxicos isolados e em sequência, principalmente nos de lábios e de língua, os indivíduos gagos não se diferenciaram dos fluentes. Deste modo, a hipótese de que o início precoce da gagueira poderia comprometer as habilidades de praxia não-verbal não se confirmou, uma vez que as habilidades investigadas não foram afetadas nos indivíduos gagos. 


\begin{abstract}
Purpose: to characterize the verbal and non-verbal praxic abilities in adult stutterers. Methods: for this research, 40 over 18-year old men and women were selected: 20 stuttering adults and 20 without communication complaints. For the praxis evaluation, they were submitted to "Protocolo de Avaliação da Apraxia Verbal e Não-verbal" (Martins and Ortiz, 2004). Results: regarding the abilities of verbal praxis, there was a statistically significant difference of number of typical and atypical disfluencies showed by the two studied groups. Phrase repetition was the only type of typical disfluency which showed a statistically significant difference between the groups. Blocking, syllable repetition and prolonging were the atypical disfluencies which showed a statistically significant difference between the groups. Regarding the non-verbal praxic abilities, a statistically significant difference was not found between the groups in the performance of lip, tongue and jaw movements, isolated and in sequence. Conclusion: the adult stutterers showed a significantly higher number of typical and atypical disfluencies than non-stutterers (verbal praxis). Stutterers do not differ from non-stutterers in their non-verbal praxic abilities. This finding contradicts those who believe that early stuttering affects non-verbal praxis.
\end{abstract}

KEYWORDS: Stuttering; Speech Disorders; Apraxias

\section{REFERÊNCIAS}

1. Suresh R, Ambrose N, Roe C, Pluzhnikov A, Wittke-Thompson JK, Thompson JK, Ng MCY, et al. New complexities in the genetics of stuttering: significant sex-specific linkage signals. Am J Hum Genet. 2006; 78(4):554-63.

2. Riaz N, Steinberg S, Ahmad J, Pluzhnikov A, Riazuddin S, Cox NJ, et al. Genomewide significant linkage to stuttering on chromosome 12. Am J Hum Genet. 2005; 76(4):647-51.

3. Viswanath N, Lee HS, Chakraborty R. Evidence for a major gene influence on persistent developmental stuttering. Hum Biol. 2004; 76(3):401-12.

4. Ludlow CL, Loucks T. Stuttering: a dynamic motor control disorder. J Fluency Disord. 2003; 28(4):273-95.

5. Andrade CRF, Cervone LM, Sassi FC. Relationship between the stuttering severity index and speech rate. São Paulo Med J. 2003; 121(2):81-4.

6. Britto Pereira MM. Análise linguística da gagueira. São Paulo: Ed. AM3 Artes, 2003 p.23.

7. Rodrigues N. Neurolinguística dos distúrbios da fala. 3. ed. São Paulo: Cortez EDUC; 1999.

8. Dewey D. What is developmental dyspraxia? Brain Cognit. 1995; 29(3):254-74.

9. Meyer PG. Tongue lip and jaw differentiation and its relationship to orofacial myofunctional treatment. Int J Orofacial Myology. 2000; 26:44-52.

10. Bloodstein O. A handbook on stuttering. San Diego: Singular; 1995.p.181-6.

11. Riley GD. Stuttering severity instrument for children and adults - SSI. Austin: Texas; 1994.
12. Andrade CRF, Zuckiewicz DV, Sassi FC. Seis parâmetros da fluência. Rev Soc Bras Fonoaudiol. 2000; 5(7):59-64.

13. Martins FC, Ortiz KZ. Proposta de protocolo para avaliação da apraxia de fala. Fono Atual. 2004; 7(30):53-61.

14. Juste F, Andrade CR. Typology of speech disruptions and grammatical classes in stuttering and fluent children. Pró-Fono. 2006; 18(2):129-40.

15. Anderson JD, Conture EG. Language abilities of children who stutter: a preliminary study. J Fluency Disord. 2005; 25(4):283-304.

16. Carlo EJ, Watson JB. Disfluencies of 3- and 5- year old Spanish-speaking children. J Fluency Disord. 2003; 28(1):37-53.

17. Max L, Caruso AJ, Gracco VL. Kinematic analyses of speech, orofacial nonspeech, and finger movements in stuttering and nonstuttering adults. J Speech Lang Hear Res. 2003; 46(1):215-32.

18. Loucks TMJ, De Nil LF, Sasisekaran J. Jawphonatory coordination in chronic developmental stuttering. J Commun Disord. 2007; 40(3):257-72.

19. Tremblay S, Shiller DM, Ostry DJ. Somatosensory basis of speech production. Nature. 2003; 423:866-9.

20. Loucks TMJ, De Nil LF. Oral kinesthetic deficit in adults who stutter: a target-accuracy study. J Motor Behav. 2006; 38(3):238-46.

21. Max L, Caruso AJ, Gracco VL. Kinematic analyses of speech, orofacial non-speech and finger movements in stuttering and non-stuttering adults. J Speech Lang Hear Res. 2003; 46(1):215-32. 
22. Max L, Gracco VL. Coordination of oral and laryngeal movements in the perceptually fluent speech of adults who stutter. J Speech Lang Hear Res. 2005; 48(3):524-42.

23. Watkins RV, Johnson BW. Language abilities in children who stutter: toward improved research and clinical applications. Lang Speech Hear Serv Sch. 2004; 35(1):82-9.

24. Subramanian A, Yairi E, Amir O. Second formant transitions in fluent speech of persistent and recovered preschool children who stutter. J Commun Disord. 2003; 36(1):59-75.

25. Subramanian A, Yairi E. Identification of traits associated with stuttering. J Commun Disord. 2006; 39(3):200-16.

26. Max L, Yudman EM. Accuracy and variability of isochronous rhythmic timing across motor systems in stuttering versus nonstuttering individuals. J Speech Lang Hear Res. 2003; 46(1):146-63.

27. Loucks TMJ, De Nil LF. Anomalous sensorimotor integration in adults who stutter: a tendon vibration study. Neurosci Letters. 2006; 402(1-2):195-200.

28. Felício CM, Freitas RLG, Vitti M, Regalo SC. Comparison of upper and lower lip muscle activity between stutterers and fluent speakers. Int $\mathrm{J}$ Pediatr Otorhinolaryngol. 2007; 71(8):1187-92.
29. Aram DM, Horwitz SJ. Sequential and nonspeech praxic abilities in developmental verbal apraxia. Dev Med Child Neurol. 1983; 25(2):197-206. 30. Farias SR, Ávila CRB, Vieira MM. Relação entre fala, tônus e praxia não-verbal do sistema estomatognático em pré-escolares. Pró-Fono. 2006; 18(3):267-76.

31. Ingham RJ, Ingham JC, Finn P, Fox PT. Towards a functional neural systems model of developmental stuttering. J Fluency Disord. 2003; 28(4):297-317. 32. Preibisch C, Raab P, Neumann K, Euler HA, Von Gudenberg AW, Gall V, et al. Event-related fMRI for the suppression of speech-associated artifacts in stuttering. Neuroimage. 2003; 19(3):1076-84.

33. Van Borsel, Achten J, Satens EP, Lahort P, Voet T. fMRI of developmental stuttering: a pilot study. Brain Lang. 2003; 85(3):369-76.

34. De Nil LF, Kroll RM, Lafaille SJ, Houle S. A positron emission tomography study of short- and long-term treatment effects on functional brain activation in adults who stutter. J Fluency Disord. 2003; 28(4):357-80.

35. Preibisch C, Neumann K, Raab P, Euler HA, Von Gudenberg AW, Lanfermann H, et al. Evidence for compensation for stuttering by the right frontal operculum. Neuroimage. 2003; 20(2):1356-64.

DOI: 10.1590/S1516-18462009005000045

RECEBIDO EM: 29/08/2008

ACEITO EM: 08/04/2009

Endereço para correspondência:

Natália Casagrande Brabo

Rua Machado, 144

Cerquilho - SP

CEP: $18520-000$

E-mail: nataliacasagrande@ @otmail.com 